\title{
TITLE:
}

\section{Results of the methodological surveys}

\section{$\operatorname{AUTHOR}(\mathrm{S})$ :}

OHGAKI, SHUN-ICHI; KOMEMOTO, KEN-ICHI; FUNAYAMA, NOBUTAKA

\section{CITATION:}

OHGAKI, SHUN-ICHI ...[et al]. 6. Results of the methodological surveys. Publications of the Seto Marine Biological Laboratory. Special Publication Series 2011, 11: 167-177

\section{ISSUE DATE:}

2011

URL:

http://hdl.handle.net/2433/159494

RIGHT: 


\section{Results of the methodological surveys}

Table 3.

The results of the survey examining the accuracy of the present method. Quadrat D3 (Table 3a) was investigated in April 2001, D8 (Table 3b) in April 2002, and D12 (Table 3c) in May 2004. For the locations of D3, D8, and D12, see Fig. 1d. In each survey, 4 or 5 researchers investigated the same quadrat by the method explained in Section 1 (overall survey, Fig. 4a), and subsequently, each of the sixty-four $1 \times 1 \mathrm{~m}$ sections in the quadrat was searched by one of the researchers (detailed survey; see Fig. 4b). In the overall survey, the maximum density $\left(\right.$ no. $\left./ \mathrm{m}^{2}\right)$ of a mollusk species in the $8 \times 8 \mathrm{~m}$ quadrats was evaluated and assigned to one of the following classes: 1 (1-9), 2 (10-99), 3 (100-999), and 4 (1000-5000). (D) indicates that only one individual was found in a quadrat. In the detailed survey, the density of each species in each section $(1 \times 1 \mathrm{~m})$ was evaluated according to the above criteria. Italic numerals in the first column indicate the species codes in the List of recorded species (Section 2). The results of the overall surveys are shown in the 2nd-5th (D3 and D8) or 2nd-6th (D12) columns, and those of the detailed survey are shown from the 7th (D3 and D8) or 8th (D12) column onward. The codes of the $1 \times 1 \mathrm{~m}$ sections (Fig. 4b) are shown in the first row. The Italic numerals in the second row indicate the researchers: (1) S. Ohgaki, (2) H. Tanase, (3) S. Yamato, (4) N. Funayama, for 2001-D3; (1) N. Funayama, (2) H. Tanase, (3) S. Ohgaki, (4) M. Sato, for 2002- D8; (1) S. Yamato, (2) N. Funayama, (3) H. Tanase, (4) K. Komemoto, (5) S. Ohgaki, for 2004-D12. +, found but density was not evaluated; -, no data. 
Figure 4.

The designs of the overall survey (a) and the detailed survey (b). In the overall survey, each researcher started from the corner (open circle) and searched for mollusk species over the entire quadrat. In the detailed survey, each researcher followed a zigzag line, recording all mollusk species encountered in each $1 \times 1 \mathrm{~m}$ section.

a

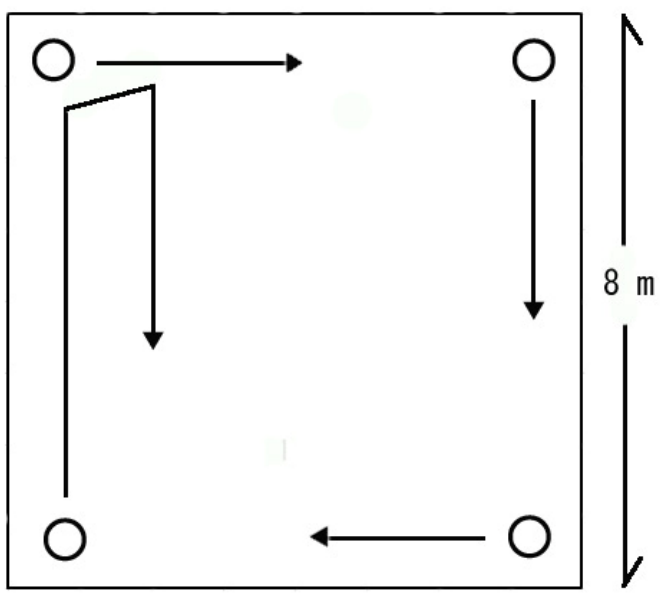

$\mathrm{b}$

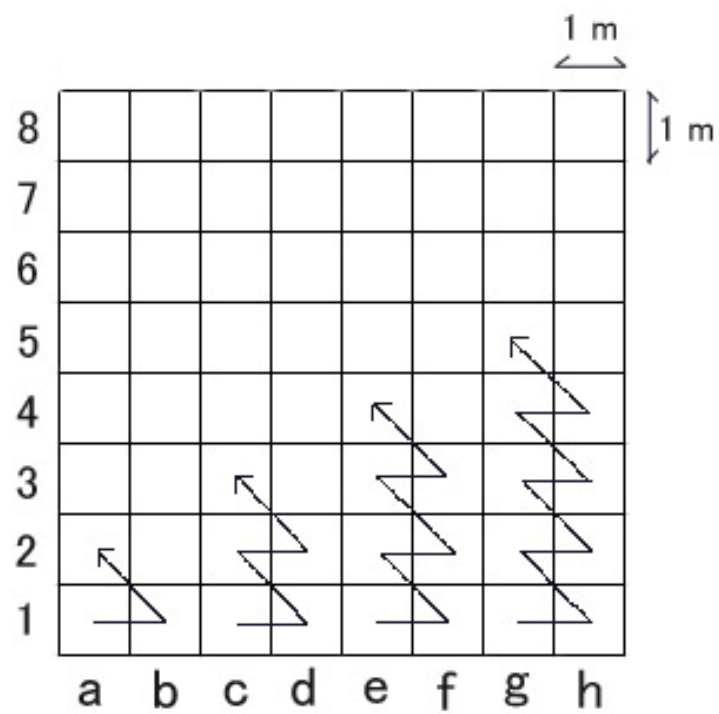




\begin{tabular}{|c|c|c|c|c|c|c|c|c|c|c|c|c|c|c|c|c|c|c|c|c|}
\hline Table $3 a-1$ & & & & & al & a2 & a3 & a4 & a5 & $a 6$ & a7 & $a 8$ & $b 1$ & $b 2$ & $b 3$ & $b 4$ & $b 5$ & $b 6$ & $b 7$ & $b 8$ \\
\hline 2001-D3 & 1 & 2 & 3 & 4 & 1 & 1 & 1 & 1 & 1 & 1 & 1 & 1 & 1 & 1 & 1 & 1 & 1 & 1 & 1 & 1 \\
\hline 4 & & & 1 & 1 & & & & & & & & & & & & & & & & \\
\hline 13 & & & & & & & & & & & & & & & & & & & & \\
\hline 15 & 1 & 2 & 1 & 2 & 1 & & 1 & & & 1 & & & & 1 & & & & & & \\
\hline 17 & (1) & & 1 & & & & & & & & & & & & & & & & & \\
\hline 20 & (1) & & & & & & & & & & & & & & & & & & & \\
\hline 28 & & & & (1) & & & & & & & & & & & & & & & & \\
\hline 44 & (1) & & 1 & (1) & & & & (1) & & & & & & & & & & & & \\
\hline 60 & 1 & & 1 & 2 & & & & & & & & & & & & & & & & \\
\hline 63 & 1 & 1 & 2 & 2 & 1 & 1 & 1 & 1 & 1 & & 1 & & 1 & & (1) & & 1 & (1) & & \\
\hline 64 & 1 & 1 & 2 & 1 & 1 & 1 & & (1) & & 1 & & & & & & & & & & \\
\hline 73 & 3 & 2 & 3 & 3 & & 2 & 3 & 2 & 2 & 2 & & 1 & 2 & 2 & 2 & 3 & 2 & & 1 & 1 \\
\hline 77 & + & 2 & + & + & & + & + & + & + & + & + & + & . & + & + & + & & + & + & \\
\hline 78 & 3 & 2 & 3 & 3 & 3 & & & (1) & & & 2 & 3 & 1 & & & 1 & 1 & 2 & 3 & 3 \\
\hline 79 & (1) & 1 & & 1 & & & & & & & & & & & & & & & & \\
\hline 81 & 2 & 2 & 3 & 2 & & & & & 1 & 1 & 1 & 2 & 1 & & 1 & 1 & (1) & 2 & 2 & 1 \\
\hline 124 & 1 & 1 & 1 & 2 & (1) & & & & & & & & 1 & & & & & & & \\
\hline 142 & (1) & & 1 & (1) & (1) & & & & & & & & & & & & & & & \\
\hline 161 & & & (1) & & & & & & & & & & & & & & & & & \\
\hline 194 & & & & (1) & & & & & & & & & & & & & & & & \\
\hline 197 & & & & (1) & & & & & & & & & & & & & & & & \\
\hline 198 & 1 & 1 & 2 & 2 & & & & & & 1 & & & & & & & & (1) & & \\
\hline 199 & & 1 & & 1 & & & & (1) & & & & & & & & & & & & \\
\hline 211 & 3 & 3 & 3 & 4 & & 2 & 2 & 2 & 3 & 3 & 3 & 3 & 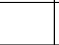 & & 2 & 3 & 3 & 3 & 3 & 3 \\
\hline 212 & 4 & & & 4 & & & & & & & & & 2 & 3 & & & & & & \\
\hline 214 & & & 1 & 1 & & (1) & & 1 & & & & & & & & & & & & \\
\hline 229 & 2 & 2 & 3 & 3 & 3 & 2 & 3 & 3 & 3 & 3 & 1 & 2 & 2 & 1. & 3 & 3 & 2 & 2 & & 2 \\
\hline 232 & + & & + & & & & & & & & & & & & & & & & & \\
\hline & & & & & & & & & & & & & & & & & & & & \\
\hline ime (min) & 30 & 41 & 43 & 30 & $a+$ & $-b=$ & $=110$ & & & & & & & & & & & & & \\
\hline
\end{tabular}




\begin{tabular}{|c|c|c|c|c|c|c|c|c|c|c|c|c|c|c|c|c|c|c|c|c|c|c|c|c|}
\hline Table $3 a-2$ & & $c 2$ & $c 3$ & $c 4$ & $c 5$ & $c 6$ & $c 7$ & $c 8$ & $d 1$ & $d 2$ & $d 3$ & $d 4$ & $d 5$ & $\frac{d 6}{d 6}$ & 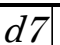 & $d 8$ & e1 & $e 2$ & $e 3$ & $e 4$ & $e 5$ & $\overline{e b}$ & $e 7$ & $e 8$ \\
\hline 2001-D3 & 2 & 2 & 2 & 2 & 2 & 2 & 2 & 2 & 2 & 2 & 2 & 2 & 2 & 2 & 2 & 2 & 3 & 3 & 3 & 3 & 3 & 3 & 3 & 3 \\
\hline 4 & & & & & & & & & & & & & & & (1) & & & & & & & & & \\
\hline 13 & 1 & & & & & & & & & & & & & & & & & & & & & & & \\
\hline 15 & 2 & 1 & & & & & & & & & & & (1) & 1 & (1) & & & & & & & & & \\
\hline 17 & 1 & 1 & & & & & & & & & & & & & & & & & & & & & & \\
\hline 20 & & & & & & & & & & & & & & & & & & & & & & & & \\
\hline 28 & & & & & & & & & & & & & & & & & & & & & & & & \\
\hline 44 & & & & & & (1) & & & & & & & & & & & & & & & & & & \\
\hline 60 & 1 & 2 & & & & & & & & (1) & & & & & & & & & & & & & & \\
\hline 63 & 2 & 2 & 1 & 2 & 1 & $x$ & & 1 & 2 & & 1 & & 1 & 1 & 2 & & & & & 1 & 1 & & 1 & \\
\hline 64 & & & (1) & 1 & 1 & 1 & & & 1 & & 1 & 1 & & (1) & & & & 2 & & 1 & 1 & & & \\
\hline 73 & 2 & 2 & 2 & 2 & 2 & 1 & 1 & 1 & 3 & 2 & & 2 & 2 & 1 & 1 & 2 & 2 & 2 & 2 & & & & 2 & 2 \\
\hline 77 & & & 2 & & 1 & 2 & 1 & 2 & 1 & 1 & 2 & 1 & 1 & 2 & 1 & 2 & + & + & + & + & + & + & + & + \\
\hline 78 & & & 2 & 2 & & 2 & 2 & 3 & 2 & & 3 & 2 & 3 & 3 & 3 & 3 & & & & 2 & 2 & 2 & 2 & 2 \\
\hline 79 & & & & & & & 1 & 1 & & & & & & & 1 & 2 & & & & & & & & \\
\hline 81 & & 1 & 2 & 1 & 1 & 1 & 1 & 1 & & 1 & & & & & 1 & & 1 & 2 & 2 & 2 & 2 & 2 & 3 & 2 \\
\hline 124 & 2 & 1 & & & & & & & & & & & & & & & & & & & & & & \\
\hline 142 & & & & & & & & & & & & & & & & & & & & & & & & \\
\hline 161 & & & & & & & & & & & & & & & & & & & & & & & (1) & \\
\hline 194 & & & & & & & & & & & & & & & & & & & & & & & & \\
\hline 197 & & & & & & & & & & & & & & & & & & & & & & & & \\
\hline 198 & & & & & & & 1 & & & & & 1 & & 1 & & & 1 & & & 1 & & & 1 & 2 \\
\hline 199 & & & & & & & & & & & & & & & & & & & & & & & & \\
\hline 211 & & & 2 & 3 & 3 & 3 & 3 & 3 & & 2 & 2 & 2 & 3 & 3 & 2 & 2 & 3 & 3 & 3 & 3 & 3 & 3 & 3 & 3 \\
\hline 212 & 3 & 3 & 2 & & & & & & & & & & & & & & & & & & & & & \\
\hline 214 & (1) & & & & & & & & & & & & & & & & & & & & & & & \\
\hline 229 & 2 & & 2 & 2 & 2 & 1 & 1 & 2 & 2 & 2 & 1 & 2 & 2 & 2 & 2 & 2 & 2 & 2 & 2 & 2 & 1 & 1 & 2 & 2 \\
\hline 232 & & & & & & & & & & & & & & & & & & & & & & & & \\
\hline & & & & & & & & & & & & & & & & & & & & & & & & \\
\hline ime $(\min )$ & $c+$ & $a=$ & 10 & & & & & & & & & & & & & & 10 & 7 & 6 & 9 & 6 & 5 & 7 & 5 \\
\hline
\end{tabular}




\begin{tabular}{|c|c|c|c|c|c|c|c|c|c|c|c|c|c|c|c|c|c|c|c|c|c|c|c|c|}
\hline Table $3 a-3$ & f1 & $f 2$ & f3 & f4 & f5 & $f 6$ & $f 7$ & $f 8$ & $g 1$ & $g 2$ & $g 3$ & $g 4$ & $g 5$ & $g 6$ & $g 7$ & $g 8$ & $h 1$ & $h 2$ & $h 3$ & h4 & $h 5$ & $h 6$ & $h 7$ & $h 8$ \\
\hline 2001-D3 & 3 & 3 & 3 & 3 & 3 & 3 & 3 & 3 & 4 & 4 & 4 & 4 & 4 & 4 & 4 & 4 & 4 & 4 & 4 & 4 & 4 & 4 & 4 & 4 \\
\hline 4 & & & & & & & & & & & & & & & & & & & & & & & & \\
\hline 13 & & & & & & & & & & & & & & & & & & & & & & & & \\
\hline 15 & & & & & & & & & & & & & & 1 & 1 & + & & & & & & & & \\
\hline 17 & & & & & & & & & & & & & & & & & & & & & & & & \\
\hline 20 & & & & & & & & & & & & & & & & & & & & & & & & \\
\hline 28 & & & & & & & & & & & & & & & & & & & & & & & & \\
\hline 44 & & & & & & & & & & & & & & & & & & & & & & & & \\
\hline 60 & & & & & & & & & & & & & & & & & & & & & & & & \\
\hline 63 & & & 1 & & & & & & & + & & & & (1) & & 1 & & & & & & & & \\
\hline 64 & 2 & 1 & 2 & 1 & & & & & 1 & 2 & 1 & 1 & & 2 & & & 1 & 1 & 1 & 1 & 1 & & & 1 \\
\hline 73 & 1 & & 1 & 2 & 2 & & 1 & 2 & 1 & 2 & 2 & & 1 & 2 & 2 & 1 & 2 & 2 & 1 & & & & 2 & 2 \\
\hline 77 & + & + & + & + & 3 & + & & & + & + & + & + & + & + & + & + & + & + & & + & + & + & + & + \\
\hline 78 & 2 & 3 & 2 & 3 & 3 & 2 & & 2 & 4 & 3 & 3 & 3 & 4 & 2 & & & 3 & & 3 & 3 & 4 & 3 & 2 & 3 \\
\hline 79 & & & & & & & & & 1 & 1 & 1 & & 1 & & & 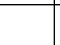 & & 1 & 1 & 1 & & & & \\
\hline 81 & 3 & 3 & 3 & 3 & 3 & 2 & 1 & 2 & 2 & 2 & 2 & 2 & 2 & 2 & 1 & 2 & 2 & 3 & 2 & 2 & 2 & 2 & 2 & 2 \\
\hline 124 & (1) & & & & & & & & & & & & & & & & & & & & & & & \\
\hline 142 & & & & & & & & & & & & & & & & & & & & & & & & \\
\hline 161 & & & & & & & & & & & & & & & & & & & & & & & & \\
\hline 194 & & & & & & & & & & & & & & & & & & (1) & & & & & & \\
\hline 197 & & & & & & & & & & & & & & & & & & & & & & & & \\
\hline 198 & & & & & & 2 & 1 & 1 & & & & 1 & & 1 & 1 & 1 & & & & & & & & \\
\hline 199 & & & & & & & & & & & & & & 1 & & & & & & & & & & \\
\hline 211 & 3 & 2 & 3 & 3 & 2 & 2 & & 2 & 4 & 3 & 3 & 3 & 3 & 3 & 2 & 2 & 3 & 3 & 3 & 4 & 4 & 3 & 3 & 3 \\
\hline 212 & & & & & & & & & & & & & & & & & & & & & & & & \\
\hline 214 & & & & & & & & & & 1 & & & & & & & & & & & & & & \\
\hline 229 & 1 & 1 & 1 & 2 & 2 & 2 & 3 & 2 & 2 & 2 & 2 & 2 & 1 & 2 & 3 & 3 & 2 & 2 & 2 & & & & 2 & 2 \\
\hline 232 & & & & & & & & & & & & & & & & & & & & & & & & \\
\hline & & & & & & & & & & & & & & & & & & & & & & & & \\
\hline ime (min) & 5 & 5 & 5 & 5 & 5 & 5 & 5 & 7 & & $g+$ & $\mathrm{h}=$ & $=11$ & & & & & & & & & & & & \\
\hline
\end{tabular}




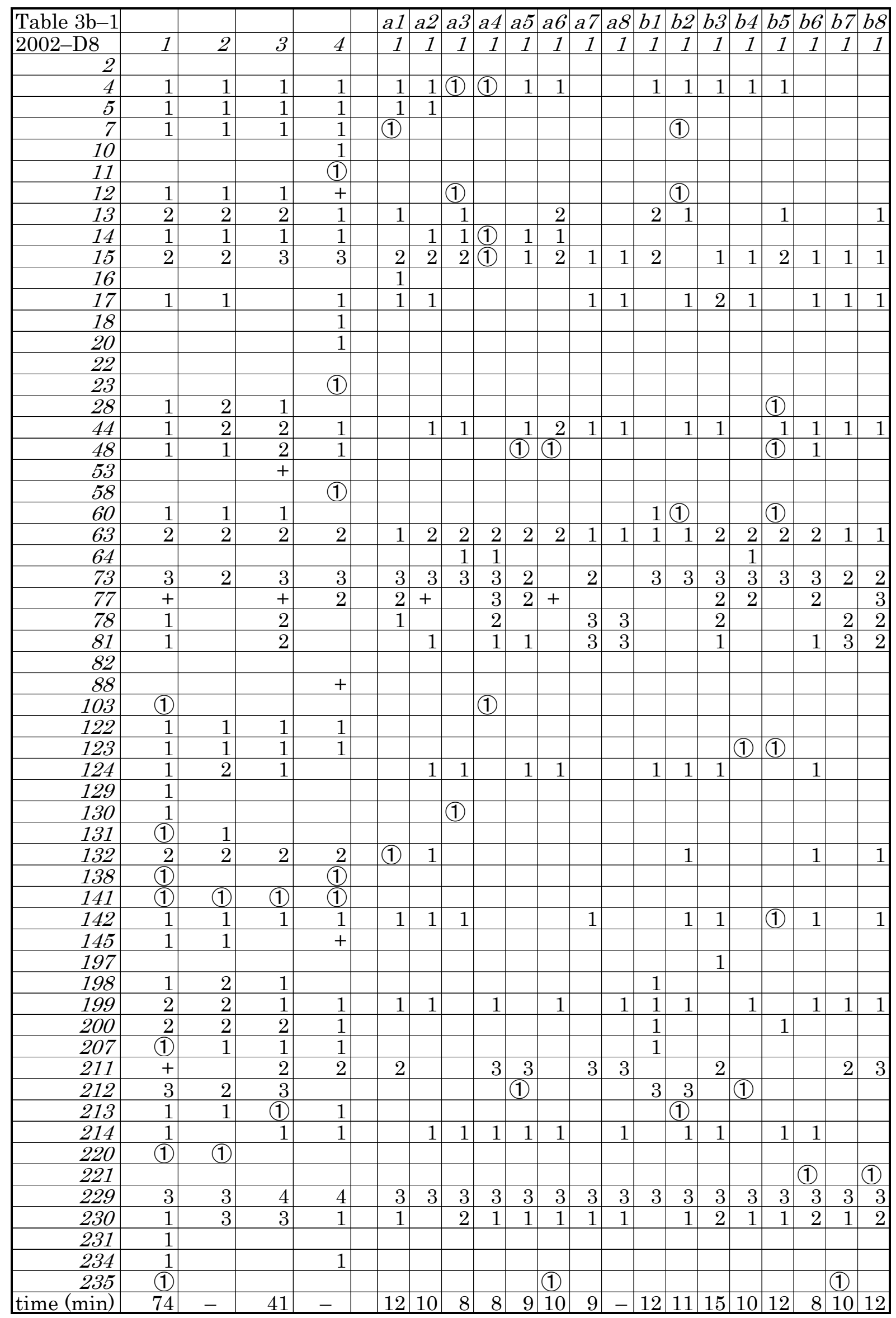




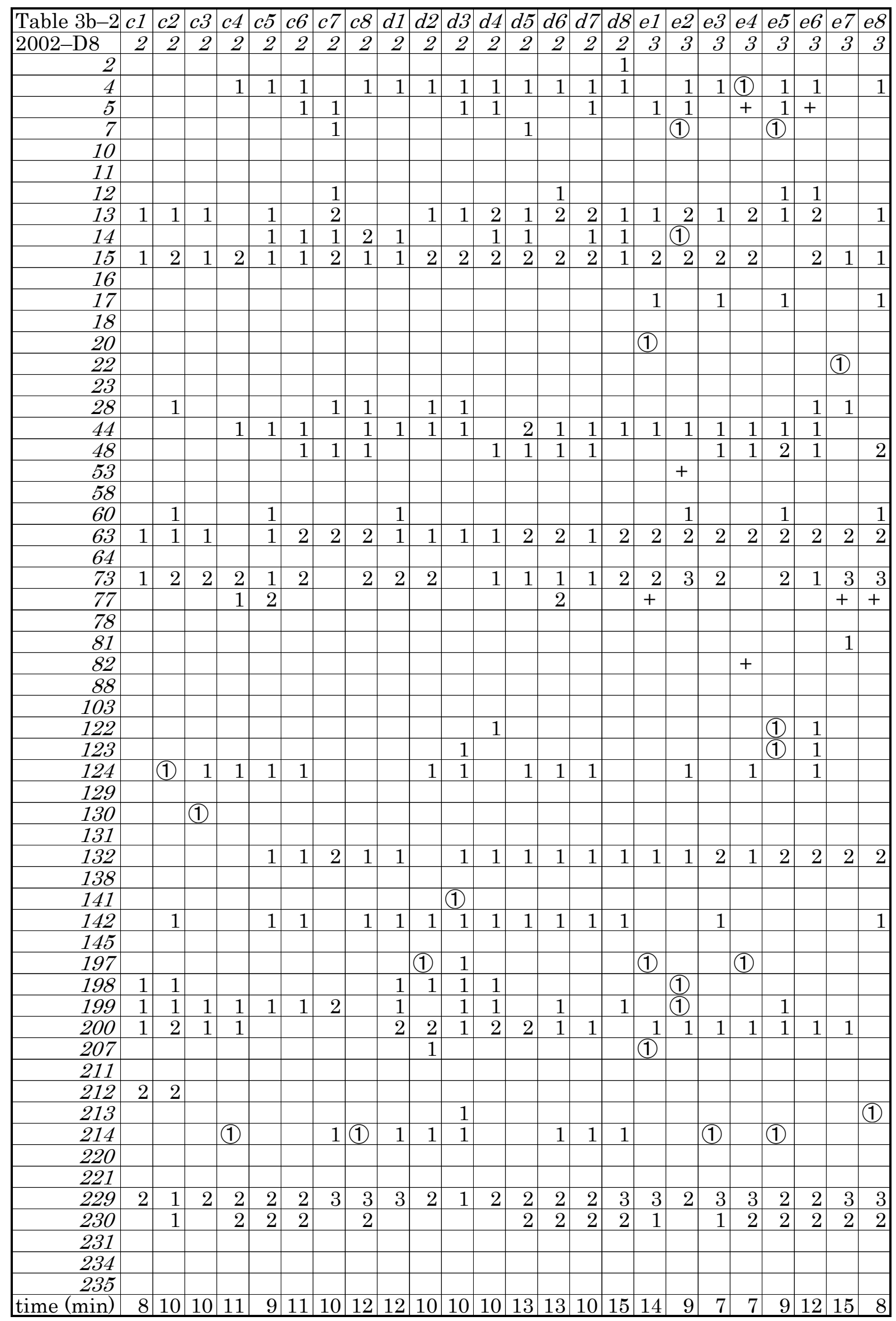




\begin{tabular}{|c|c|c|c|c|c|c|c|c|c|c|c|c|c|c|c|c|c|c|c|c|c|c|c|c|c|}
\hline Table $3 \mathrm{~b}-3$ & & $f 2$ & f3 & $f 4$ & $f 5$ & $f 6$ & $f 7$ & $f 8$ & $g$ & 1 & 2. & g3 & $g 4$ & $g 5$ & $g 6$ & $g 7$ & $g 8$ & h1 & h2 & h3. & $h 4$ & $h 5$ & $h 6$ & h7 & $h 8$ \\
\hline 2002-D8 & 3 & 3 & 3 & 3 & 3 & 3 & 3 & 3 & & 4 & 4 & 4 & 4 & 4 & 4 & 4 & 4 & 4 & 4 & 4 & 4 & 4 & 4 & 4 & 4 \\
\hline & & & & & & & & & & & & & & & & & & & & & & & & & \\
\hline 4 & 1 & 1 & & 1 & 1 & 1 & & & 1 & & 1 & 1 & (1) & (1) & (1) & & 1 & & 1 & 1 & & (1) & 1 & 1 & 1 \\
\hline 5 & & & & & 1 & 1 & & & & 1 & 1 & 1 & 1 & 1 & 1 & 1 & & 1 & & 1 & & & & 1 & 1 \\
\hline 7 & & (1) & & & & & & & & & & 1 & (1) & 1 & & 1 & & & & & (1) & & (1) & & \\
\hline 10 & & & & 1 & & & & & & & & & (1) & (1) & 1 & & 1 & & & & & 1 & & & (1) \\
\hline 11 & & & & & & & & & & 1 & 1 & & 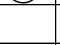 & (1) & & (1) & (1) & & & (1) & (1) & & & & \\
\hline 12 & & & & & & & & & (1) & D & 1 & (1) & & & & & & & (1) & & & & & & \\
\hline 13 & 1 & & (1) & 1 & 1 & 1 & 1 & 1 & & & 1 & 1 & & 1 & 1 & 1 & 1 & 1 & & 1 & (1) & 1 & & 1 & 1 \\
\hline 14 & & & & & & & & & & 1 & 1 & 1 & 1 & & & 1 & 1 & 1 & 1 & & & & 1 & 1 & 1 \\
\hline 15 & & 1 & 1 & 2 & 1 & & 2 & 2 & & 1 & 1 & 1 & 1 & 2 & 2 & 1 & 1 & 1 & & 2 & & 2 & 1 & 1 & 1 \\
\hline 16 & & & & & & & & & & & & & & & & & & & & & & & & 1 & 1 \\
\hline 17 & 1 & & & & 1 & & 1 & & & 1 & & 1 & 1 & 1 & 1 & 1 & 1 & 1 & 1 & & & 1 & & 1 & 1 \\
\hline 18 & & & & & & & & & & & & & & & & & & & & & & (1) & & & \\
\hline 20 & & & & & & & & & (1) & D & & & (1) & & & & & 1 & & & & & & & \\
\hline 22 & & & & & & & & & & & & & & & & & & & & & & & & & \\
\hline 23 & & & & & & & & & & & 1 & & & & & & & 1 & 1 & & & & & & \\
\hline 28 & & & & & & & (1) & 1 & & & & & & & & & & & & & & & & & \\
\hline 44 & & 1 & 1 & 2 & & & 1 & (1) & & 1 & 2 & 2 & 1 & & 1 & 1 & 1 & 1 & 2 & & & 1 & 1 & & \\
\hline 48 & & & 1 & 1 & & & & & & & (1) & 1 & & & & & & & & & & & & & \\
\hline 53 & & & & + & & & & & & & & & & & & & & & & & & & & & \\
\hline 58 & & & & & & & & & & & & & & & & (1) & & & & & & & & & \\
\hline 60 & & & & & & & & & & & & & (1) & & & & (1) & & & (1) & & (1) & & (1) & (1) \\
\hline 63 & 1 & 1 & 1 & 1 & 1 & (1) & 1 & 1 & & 2 & 2 & 2 & 2 & 1 & 1 & 2 & 2 & 2 & 2 & 1 & 1 & 1 & 1 & 1 & 1 \\
\hline 64 & & & & & & & & & & & & & & & & & & & & & & & & & \\
\hline 73 & 2 & & 2 & 2 & 2 & & 2 & 2 & & 3 & 3 & 3 & 3 & 2 & 2 & 2 & 3 & 4 & 3 & 2 & & 2 & 1 & 2 & 2 \\
\hline 77 & & + & & & & & & & & 2 & 2 & 2 & 2 & & & 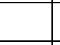 & 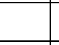 & 2 & 2 & & & & & 3 & \\
\hline 78 & & & & & & & & & & & & & & & & & & & & & & & & & \\
\hline 81 & & & & & & & & & & & & & & & & & & & & & & & & & \\
\hline 82 & & & & & & & & & & & & 1 & & & & 1 & & & & & & & & & \\
\hline 88 & & & & & & & & & & & & & & & & & & & & & & & & 1 & \\
\hline 103 & & & & & & & & & & & & & & & & & & & & & & & & & \\
\hline 122 & & & & (1) & (1) & (1) & (1) & & & & 1 & 1 & 2 & 1 & & 1 & & & & 1 & 1 & (1) & 1 & 2 & 1 \\
\hline 123 & & & & (1) & 1 & 1 & 1 & 1 & 1 & D) & 1 & 1 & (1) & 1 & 1 & 1 & & & (1) & 1 & 1 & 1 & 2 & 1 & \\
\hline 124 & 1 & 1 & & 1 & & & & & & & & & & & & & & & & & & & & & \\
\hline 129 & & & & & & & & & & & & & & & & & & & & & & & & & \\
\hline 130 & & & & & & & (1) & & & & & & & & & & & & & & & & & & \\
\hline 131 & & & & & & & & (1) & & & & & & & & & & & & & & & & & \\
\hline 132 & 2 & 2 & 2 & 2 & 2 & 2 & 2 & 2 & & 2 & 2 & 2 & 2 & 2 & 2 & 2 & 1 & 1 & 1 & 1 & 1 & 2 & 1 & 2 & 2 \\
\hline 138 & & & & & & & & & & & & & & & & & & & & & & & (1) & & \\
\hline 141 & & & & & & & & & & & & & & & & & & & & & & & & 1 & \\
\hline 142 & & & 1 & 1 & & 1 & 1 & 1 & & 1 & 1 & 1 & 1 & 1 & & 1 & 1 & 1 & 1 & & & 1 & 1 & & 1 \\
\hline 145 & & & & & 1 & 1 & & & & & & & & & & & & & & 1 & 1 & & & & \\
\hline 197 & & & & & & & & & & & & & & & & & & & & & & & & & \\
\hline 198 & & & & & & & & & & & & & & & & & & & & & & & & & \\
\hline 199 & & & 1 & & & & & & & & 1 & 1 & & (1) & (1) & (1) & 1 & & & (1) & & (1) & & & (1) \\
\hline 200 & & 1 & & & (1) & & 1 & 1 & & & & & & 1 & 1 & 1 & & & & & & 1 & & & \\
\hline 207 & (1) & & & & & & & & & & & & 1 & 1 & (1) & & & & & & (1) & (1) & & & (1) \\
\hline 211 & & & & & & & & & & 1 & 2 & 2 & 2 & & & & & & & & & & & & 2 \\
\hline 212 & & & & & & & & & & & & & & & & & & & & & & & & & \\
\hline 213 & & & & & & 1 & & & & & & 1 & (1) & 1 & & 1 & & & & 1 & 1 & & 1 & 1 & 1 \\
\hline 214 & & & & & & & & & & & & & (1) & (1) & & & & & & & & & & & \\
\hline 220 & & & & & & & & & & & & & & & & & & & & & & & & & \\
\hline 221 & & & & & & & & & & & & & & & & & & & & & & & & & \\
\hline 229 & 3 & 3 & 2 & 3 & 2 & 1 & 2 & 2 & & 3 & 3 & 3 & 3 & 2 & 1 & & 3 & 2 & 2 & & & 2 & 2 & 2 & 3 \\
\hline 230 & 1 & 1 & 2 & 2 & 1 & 2 & 2 & 2 & & 1 & 1 & 1 & 1 & 1 & 2 & 2 & 3 & 2 & 2 & 1 & 1 & 1 & 1 & 2 & 3 \\
\hline 231 & & & & & & (1) & & & & & & & & & & & & & & 1 & & & (1) & 1 & \\
\hline 234 & & & & & & & & & & & & & & & & & & & & (1) & (1) & & & 1 & \\
\hline 235 & & & & & & & & & & & & & & & & & & & & & & & & & \\
\hline ime $(\min )$ & 13 & 10 & 8 & 9 & 14 & 9 & 10 & 10 & & 9 & 15 & 17 & 11 & 7 & 7 & 15 & 11 & 10 & 7 & 24 & 7 & 12 & 11 & 11 & 8 \\
\hline
\end{tabular}




\begin{tabular}{|c|c|c|c|c|c|c|c|c|c|c|c|c|c|c|c|c|c|c|c|c|c|c|c|}
\hline Table $3 \mathrm{c}-1$ & & & & & & $a 1$ & & $2 a_{c}$ & & $\overline{a 4}$ & & & & $\overline{a 7}$ & $a 8$ & $b 1$ & $b 2$ & $b 3$ & $b 4$ & $b 5$ & $b 6$ & $b 7$ & $b 8$ \\
\hline 2004-D12 & 1 & 2 & 3 & 4 & 5 & 1 & & 7 & 1 & 1 & t. & & 5 & 5 & 5 & 1 & 1 & 1 & 1 & 1 & 1 & 5 & 5 \\
\hline 3 & (1) & & 1 & 1 & & & & & & & & & & & & & & & & & & & \\
\hline 4 & 1 & 2 & 1 & 1 & 2 & 1 & & 1 & & 2 & & 2 & 1 & 1 & & 2 & & 1 & 2 & 2 & & 1 & 1 \\
\hline 5 & 1 & 2 & 1 & & 1 & 1 & & & & 1 & & 1 & & & & & 1 & 1 & & 1 & 1 & & \\
\hline 6 & & & & & & & & & & & & & & & & & & & & & & & \\
\hline 7 & 1 & 1 & 1 & 1 & 1 & 1 & & 1 & & & & 1 & & 1 & 1 & 1 & 1 & & 1 & & & & \\
\hline 8 & (1) & & & & & & & 2 & 1 & & & & & & & & & 1 & 1 & & & & \\
\hline 10 & 1 & 1 & 1 & 1 & 2 & & 1 & & 1 & & & & 2 & 2 & 2 & & & & & & & 3 & 3 \\
\hline 13 & 1 & 1 & 2 & 1 & 1 & 1 & & 1 & 1. & & & 1 & & & & 1 & & & & & & 1 & 1 \\
\hline 14 & & & & & & & & & & & & & & & & & & & & & & & \\
\hline 15 & 1 & 2 & & 2 & 2 & 1 & & 2 & 2 & 2 & & 3 & 1 & . & & 2 & 1 & 2 & 2 & & 2 & 2 & 1 \\
\hline 16 & 2 & 1 & 1 & 2 & 2 & & & 2 & 1 & 1 & & & 1 & 1 & 1 & 1 & 2 & 1 & & + & 1 & & \\
\hline 17 & 1 & 2 & 1 & 1 & 1 & 1 & & 2 & 1 & 1 & & 1 & 1 & 1 & (1) & 2 & 1 & 1 & 1 & + & 1 & & 1 \\
\hline 18 & 1 & & & & & & & & & & & & & 1 & & & & & & & & & \\
\hline 26 & (1) & 1 & (1) & & & & & & & & & & & & 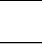 & & & & & & & & \\
\hline 28 & 1 & 2 & 1 & 1 & 1 & & & & & & 1 & & 1 & 1 & & & & & & 2 & & 1 & \\
\hline 48 & 2 & 2 & 2 & 2 & 2 & 2 & 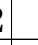 & 2 & 2 & 2 & & 2 & 2 & 2 & 2 & 2 & 2 & 2 & 3 & 2 & 2 & 2 & 2 \\
\hline 53 & & & & & + & & & & & & & & & (1) & & & & & & & & & \\
\hline 77 & & & 2 & & & & & & & & & & & & & & & & & & & & \\
\hline 87 & 1 & 1 & & & 1 & & & & & & & & & & & & & & & & & 1 & 1 \\
\hline 88 & & 1 & & (1) & & & & & & & & & & & & & & & & & & & \\
\hline 94 & & & (1) & (1) & (1) & & & & & & & & & & & & & & & & & & \\
\hline 123 & & (1) & & & 1 & & & & & & & & & & & & & & & & & & \\
\hline 124 & & & & & & & & & & & & & & & & & & & & & & & \\
\hline 129 & (1) & (1) & & & & & & & & & & & & & & & & & & & & & \\
\hline 130 & 1 & 1 & 1 & 1 & 1 & & & 1 & 1 & & & & & & & & (1) & & & & & & \\
\hline 131 & & & & & & & & & & & & & & & & & & & & & & & \\
\hline 132 & 2 & 1 & 1 & 1 & 2 & & & & & 1 & & & & & & & (1) & (1) & 1 & 1 & & & \\
\hline 141 & 1 & 1 & & (1) & 1 & 1 & & 1 & 1 & 1 & & & & & & 1 & & 1 & (1) & & & & \\
\hline 142 & 2 & 1 & 1 & 2 & 1 & 1 & & 1 & & 1 & & & & & & 1 & & 1 & 1 & + & & (1) & 1 \\
\hline 143 & & & & 1 & (1) & & & & & & & & & & & & & & & & & & \\
\hline 144 & (1) & (1) & (1) & & & & & & & & & & & & & & & & & & & & \\
\hline 145 & (1) & 1 & (1) & & & & & & & & & & & & & & & & & & & & \\
\hline 148 & & (1) & & & & & & & & & & & & & & & & & & & & & \\
\hline 176 & & & & & & & & & & & & & & & & & & & & & & (1) & \\
\hline 199 & & 1 & & & 1 & & & & & & & & & & & & & & & & & & \\
\hline 200 & 2 & 1 & 2 & 2 & 2 & & & 2 & 1 & & & 1 & & 1 & 1 & & 2 & 1 & & & 2 & 1 & 2 \\
\hline 202 & & & & & & & & & & & & & & & & & & & & & & & \\
\hline 207 & 2 & 1 & 1 & 1 & 1 & & & 1 & & & & & & & & 1 & 1 & & & & & & (1) \\
\hline 212 & 4 & 4 & 3 & 4 & 4 & 3 & & 3 & 3 & 1 & & 3 & 4 & 4 & 4 & 3 & 3 & 3 & 2 & & 3 & 4 & 2 \\
\hline 213 & 2 & 1 & 1 & 1 & 1 & & (1) & & & & & & & & & & & & 1 & 1 & & (1) & \\
\hline 214 & 3 & & 1 & 1 & 3 & 3 & & 2 & 1 & 2 & & 2 & 2 & 2 & & 3 & 2 & 3 & 2 & 1 & 1 & & \\
\hline 216 & & & & & & & & & & & & & & & & & & & & & & & \\
\hline 220 & & (1) & & & & & & & & & & & & & & & & & & & & & \\
\hline 222 & & (1) & & (1) & & 1 & & & & & & & & & & & & & & & & & \\
\hline 229 & 2 & 2 & 2 & 2 & 2 & 2 & 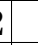 & 1 & 2 & 2 & & 2 & 2 & 2 & 2 & 2 & 2 & 2 & 1 & 1 & 2 & 2 & 2 \\
\hline 230 & 3 & 2 & 2 & 2 & 2 & 1 & & & & 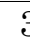 & & 2 & 1 & 1 & & 2 & 1 & 1 & 3 & 2 & 2 & (1) & (1) \\
\hline 233 & & & & & & & & & & & & & & & & & & & & & (1) & & \\
\hline 234 & & & & & & & & & & & & & & & & & & & & & & & \\
\hline 235 & & & & & & & & & & & & & & & & & & & & & & & \\
\hline & & & & & & & & & & & & & & & & & & & & & & & \\
\hline time (min) & 52 & 55 & 50 & 42 & 38 & 11 & 1 & & 12 & $\Phi$ & & \begin{tabular}{l|l}
0 \\
\end{tabular} & 7 & 6 & 5 & 10 & 10 & 9 & 9 & 9 & 9 & 6 & 5 \\
\hline
\end{tabular}




\begin{tabular}{|c|c|c|c|c|c|c|c|c|c|c|c|c|c|c|c|c|c|c|c|c|c|c|c|c|}
\hline Table $3 c-2$ & $c 1$ & $c 2$ & $c 3$ & $c 4$ & $c 5$ & $c 6$ & $c 7$ & $c 8$ & $d 1$ & $d 2$ & $d 3$ & $d 4$ & $d 5$ & $d 6$ & $d 7$ & $d 8$ & e1 & $e 2$ & $e 3$ & $e 4$ & $e 5$ & $e 6$ & $e 7$ & $e 8$ \\
\hline 2004-D12 & 2 & 2 & 2 & 2 & 2 & 2 & 5 & 5 & 2 & 2 & 2 & 2 & 2 & 2 & 5 & 5 & 3 & 3 & 3 & 3 & 3 & 3 & 5 & 5 \\
\hline 3 & & & & & & & & & & & & & & & & & & & & & & (1) & & \\
\hline 4 & 2 & 1 & & 1 & & 1 & 2 & 1 & 1 & 1 & & 2 & 1 & & 2 & & & 1 & & 1 & & 1 & 1 & \\
\hline 5 & & & 1 & 1 & & & 1 & & & & & 1 & & & (1) & & 1 & 1 & 1 & 1 & 1 & & & \\
\hline 6 & & & & & & & & & & & & & & & & & & & & & & (1) & & \\
\hline 7 & 1 & & (1) & 1 & 1 & 1 & 1 & 1 & & (1) & & 1 & 1 & 1 & 1 & & & & 1 & 1 & & 1 & & \\
\hline 8 & & & & & (1) & (1) & & & & & & & & & & & & & & & & & & \\
\hline 10 & & & 1 & & & & & 2 & & & 1 & & & & 2 & 2 & & & & & & 1 & 1 & 1 \\
\hline 13 & 1 & 1 & 1 & & 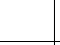 & & & (1) & 1 & & 1 & & & & & & & & & & & & & \\
\hline 14 & & & & & & & & & & & & & & & & & & & & & & & & \\
\hline 15 & 1 & 2 & & 2 & 2 & & 2 & & 1 & 1 & & 2 & & & 1 & & 2 & 1 & 2 & & & 2 & 1 & \\
\hline 16 & 2 & & & & & & 1 & 1 & 1 & 1 & 1 & & & & 1 & 1 & & 1 & 1 & & 1 & & & \\
\hline 17 & 2 & 1 & & & 1 & & & & & & & & & & & & 1 & 1 & 1 & & & & & \\
\hline 18 & & & & & & & & & & & & & & & & & & & & & & & & \\
\hline 26 & & & & & & 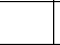 & & & & & & & & & & & & & & & & (1) & & \\
\hline 28 & 1 & 1 & 1 & 1 & 2 & & & & & & 1 & 1 & 2 & 1 & & & & & 1 & & 1 & & & \\
\hline 48 & 2 & 2 & 2 & 2 & 2 & 2 & 2 & 2 & 2 & 2 & 2 & & 1 & & & & 1 & 2 & 1 & & & & 1 & \\
\hline 53 & & & & & & & & & & & & & & & & & & & & & & & & \\
\hline 77 & & & & & & & & & & & & & & & & & & & & & & & & \\
\hline 87 & & & & & & & & 1 & & & & & & & (1) & & & & & & & & (1) & \\
\hline 88 & & & & & & & & & & & & & & & (1) & (1) & & & & & & & & \\
\hline 94 & & & & & & & & & & & & & & & & & & & & & & & & \\
\hline 123 & & & & & & & & & & & & & & & & & & & & & & 1 & 1 & \\
\hline 124 & & & & & & & & & & & & & & & & & & & & & & & & \\
\hline 129 & & & & & & & & & & & & & & & & & & & & & & & & \\
\hline 130 & (1) & & (1) & & & & & & (1) & & & (1) & & 1 & & & & & & & & 1 & 1 & 1 \\
\hline 131 & & & & & & & & & & & & & & & & & & & & & & & & \\
\hline 132 & & + & 2 & 2 & 1 & 1 & 1 & 1 & & & 2 & 2 & 2 & 2 & 2 & 2 & & & (1) & 1 & 1 & 2 & 1 & 2 \\
\hline 141 & & 1 & 1 & 1 & & & & & & 1 & 1 & & 1 & 1 & & & & & & & & & & 1 \\
\hline 142 & 1 & 1 & 1 & 1 & (1) & & (1) & & & & & 1 & & & 2 & 1 & 1 & & 1 & 1 & & 1 & 1 & 2 \\
\hline 143 & & & & (1) & & & & & & & & & & & & & & & & & & & & \\
\hline 144 & & & & & & & & & & & & & & & & & & & & & & & & \\
\hline 145 & & & & & & & & & & & & & & & & & & & & & & & & \\
\hline 148 & & & & & & & & & & & & & & & & & & & & & & & & \\
\hline 176 & & & & & & & & & & & & & & & & & & & & & & & & \\
\hline 199 & & & & & & & (1) & & & & & & & & & & & & 2 & & & & & \\
\hline 200 & & 1 & & & & & & 1 & & 1 & & 1 & & 2 & 1 & & 1 & & & & 1 & 2 & 1 & \\
\hline 202 & & & & & & & & & & & & & & 1 & & & & & & & & & & \\
\hline 207 & & (1) & (1) & & & & 1 & & & (1) & 1 & 1 & 1 & 2 & 1 & (1) & & & (1) & 1 & 1 & & 1 & \\
\hline 212 & 4 & 4 & 4 & 4 & 3 & 3 & 3 & & 4 & 4 & 4 & 4 & 4 & 3 & 4 & 3 & 3 & 4 & 4 & 3 & 3 & 3 & 3 & 4 \\
\hline 213 & 1 & (1) & 1 & 1 & 1 & & (1) & & 1 & & 1 & 1 & 1 & 1 & (1) & & & & 1 & & 1 & & (1) & \\
\hline 214 & 2 & 2 & 2 & 3 & 2 & 2 & & & & 3 & 2 & & 2 & 2 & & & 1 & 1 & & & & & & \\
\hline 216 & & & & & & & & & & & & (1) & & & & & & & & & & & & \\
\hline 220 & & & & & & & & & & & & & & & & & & & & & & & & \\
\hline 222 & & & & & & & & (1) & (1) & & & & & & & & & & & & & & & \\
\hline 229 & 2 & 2 & 2 & 2 & 2 & 2 & 2 & 1 & 2 & 2 & 2 & 2 & 2 & 2 & 2 & 1 & & & 1 & & & & & \\
\hline 230 & 2 & 2 & 2 & & 1 & 1 & 1 & 1 & 2 & 2 & 1 & 1 & & & & & 1 & 2 & & & & & & \\
\hline 233 & & & & & & & & & & & & & & & & & & & & & & & & \\
\hline 234 & & & & & & & & & & & & & & & & & & & & & & & & \\
\hline 235 & & & & & & & & & & & & & & & & & & & & & & & & \\
\hline & & & & & & & & & & & & & & & & & & & & & & & & \\
\hline ime (min) & 13 & 7 & 6 & 7 & 10 & 6 & 7 & 5 & 9 & 7 & 9 & - & 7 & 8 & 7 & 6 & 10 & 5 & & 10 & 7 & 9 & & 8 \\
\hline
\end{tabular}




\begin{tabular}{|c|c|c|c|c|c|c|c|c|c|c|c|c|c|c|c|c|c|c|c|c|c|c|c|c|c|}
\hline Table $3 c-3$ & $f 1$ & $f 2$ & $f 3$ & $f 4$ & $f 5$ & $f 6$ & $f 7$ & $f 8$ & $g$ & & $2 z_{z}$ & o3 & $g 4$ & $g 5$ & $g 6$ & $g 7$ & $g 8$ & h1 & h2 & h3. & h4. & h5. & $h 6$ & $h 7$ & $\overline{h 8}$ \\
\hline 2004-D12 & 3 & 3 & 3 & 3 & 3 & 3 & 3 & 5 & 5 & & 4 & 4 & 4 & 4 & 4 & 4 & 5 & 4 & 4 & 4 & 4 & 4 & 4 & 4 & 5 \\
\hline 3 & & & & & & (1) & & & & & & & & & & & & & & & & & & & \\
\hline 4 & & 1 & 1 & 1 & 1 & 1 & 1 & & & L & 1 & 2 & 2 & 2 & 2 & 2 & 1 & 1 & 1 & 2 & 2 & 2 & 2 & 2 & 1 \\
\hline 5 & & 1 & & 1 & 1 & 1 & & & & & 1 & & & 1 & (1) & (1) & 1 & 1 & 1 & 1 & 1 & & 1 & & \\
\hline 6 & & & & & & & & & & & & & & & & & & & & & & & & & 1 \\
\hline 7 & 1 & & 1 & & & & 1 & & & L & 1 & 1 & (1) & & (1) & 1 & 1 & & & & & & (1) & 1 & 1 \\
\hline 8 & & & & & & & & & & & & & & & & & & & & & & & & & \\
\hline 10 & 1 & 1 & & & & & & & & & 1 & & & & & 2 & 2 & 1 & & & 1 & & & & 2 \\
\hline 13 & & & & & & (1) & 1 & & 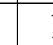 & 1 & & . & & & 1 & & & 1 & & & 1 & 1 & 1 & & \\
\hline 14 & & & & & & & & & & & & & & 2 & & & & & & & 1 & & & & \\
\hline 15 & & & 1 & & 1 & 1 & & & & L & 1 & 2 & 2 & 2 & & 1 & & 1 & 1 & 2 & 2 & 2 & 2 & 1 & 2 \\
\hline 16 & 1 & 2 & 1 & 1 & 1 & 1 & & & . & L & 1 & & 1 & & 1 & & 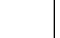 & 1 & 1 & 1 & & 1 & 1 & 2 & 1 \\
\hline 17 & 1 & 1 & 1 & 1 & & & & (1) & . & 1 & 1 & 1 & 2 & 1 & 2 & & ( & & & 2 & 2 & & 2 & & \\
\hline 18 & & & & & & & & & . & $\mathrm{L}$ & & & & & & & & & & & & & & & \\
\hline 26 & & & & & & & 1 & & & & & & & & & & & & & & & & & & \\
\hline 28 & 1 & 1 & 1 & 1 & & 1 & 1 & & & 1 & 1 & 1 & 1 & 2 & 1 & 1 & & & 1 & 1 & 1 & 1 & 1 & & 1 \\
\hline 48 & 1 & 2 & 2 & 2 & 2 & 2 & 1 & & & 1 & 1 & 1 & & 2 & 2 & 2 & 1 & 1 & 1 & 1 & 2 & 2 & 2 & 1 & 2 \\
\hline 53 & & & & & & & & & & & & & & & & & & & & & & & & & \\
\hline 77 & & & & & & & & & & & & & & & & & & & & & & & & & \\
\hline 87 & & & & & & & & & & & & & & & & & & & & & & & & & \\
\hline 88 & & & & & & & & & & & & & & & & & & & & & & & & & \\
\hline 94 & & & & & & & & & & & & & & & & & & & & & & & & & \\
\hline 123 & & & & & & & & & & & & & & & & & & & & & & & & & \\
\hline 124 & & & & & & & & & & & 1 & & & & & & & 1 & & & & & & & \\
\hline 129 & & & & & & & & & & & & & & & & & & & & & & & & & \\
\hline 130 & 1 & & & & & & 1 & 1 & & & & & & & & & 1 & & & & & & & & 1 \\
\hline 131 & & & & & & & & & & & & & & & & & & & (1) & & & & & & \\
\hline 132 & 1 & & 1 & & 1 & 1 & 1 & 2 & 2 & 2 & 1 & 1 & 1 & 1 & 1 & 1 & 1 & 1 & 2 & & 1 & 1 & 1 & & 2 \\
\hline 141 & & & & & & & & 1 & 1 & & & & & & & & & & & & & & & & \\
\hline 142 & & 1 & & & & & 1 & 1 & & & 1 & 1 & & & 1 & & 1 & 1 & 2 & & 1 & & & & 1 \\
\hline 143 & & & & & & & & & & & & (1) & & & & & & 1 & & & & & & & \\
\hline 144 & & & & & & & & & & & & & & & & & & & & & & & & & \\
\hline 145 & & & & & & & & & & & & & & & & & & & (1) & & & & & & \\
\hline 148 & & & & & & & & & & & & & & & & & & & & & & & & & \\
\hline 176 & & & & & & & & & & & & & & & & & & & & & & & & & \\
\hline 199 & & & & 1 & & & & & & & & & & & & & 1 & 1 & & & & & 1 & 1 & \\
\hline 200 & 1 & & (1) & 1 & 2 & 2 & 2 & & & L & 1 & 1 & 2 & 2 & 1 & 1 & 1 & 1 & 2 & & 2 & 2 & 2 & 1 & 1 \\
\hline 202 & & & & & & & & & & & & & & & & & & & & & & & & & \\
\hline 207 & & & 1 & & & 1 & 1 & & & & D & 1 & & 1 & & 1 & (1) & & & & & & 1 & 1 & 1 \\
\hline 212 & 1 & 4 & 3 & 3 & 3 & 1 & 2 & 3 & 3 & 3 & 3 & 1 & & & & & 3 & 4 & 3 & 3 & & & & & \\
\hline 213 & 1 & & 1 & & 1 & & & (1) & & I & & 1 & 1 & & & & 1 & 1 & 1 & 1 & & (1) & (1) & & (1) \\
\hline 214 & & & & & & & & & & L & 1 & 1 & 2 & 1 & & & & 1 & & 1 & 1 & 1 & & & \\
\hline 216 & & & & & & & & & & & & & & & & & & & & & & & & & \\
\hline 220 & & & & & & & & & & & & & & & & & & & & & & & & & \\
\hline 222 & & & & & & & & & & & & & & & & & & & & & & & & & \\
\hline 229 & 1 & & & & 2 & & & & & 2 & 2 & 2 & 2 & 1 & 1 & 1 & 1 & 2 & 1 & & & & 1 & 2 & 1 \\
\hline 230 & 2 & 2 & & 2 & 2 & 1 & 1 & & & 2 & 2 & 2 & 2 & 2 & 2 & 2 & & 2 & 2 & 2 & 2 & 1 & 2 & 2 & 1 \\
\hline 233 & & & & & & & & & & & & & & & & & & & & & & & & & \\
\hline 234 & & & & & & & & & & & & & & (1) & & 1 & & & & & & & (1) & & \\
\hline 235 & & & & & & & & & & & & & & & & & (1) & & & & & & & & \\
\hline & & & & & & & & & & & & & & & & & & & & & & & & & \\
\hline me (min) & 10 & - & 10 & 10 & 6 & 14 & 8 & 6 & & & 8 & 8 & 6 & 6 & 6 & 8 & 8 & 8 & - & 6 & 11 & 6 & 7 & 7 & - \\
\hline
\end{tabular}

